\title{
STAPHYLOCOCCUS AUREUS INFECTIONS AND THEIR ANTIBIOTIC SUSCEPTIBILITY PROFILE AT A TERTIARY CARE HOSPITAL
}

\section{ANTHONY N OFULAH, SHASHIDHAR VISHWANATH*, BARNINI BANERJEE, KIRAN CHAWLA}

Department of Microbiology, Kasturba Medical College, Manipal University, Manipal, Karnataka. India. Email: drshashidharv@gmail.com Received: 09 August 2017, Revised and Accepted: 29 September 2017

\section{ABSTRACT}

Objective: Staphylococcus aureus remains an important bacterial pathogen causing diverse infections which are both nosocomial and community acquired. Increasing resistance among $S$. aureus to various antibiotics is a cause of concern.

Methods: A prospective observational study of 4 months duration was conducted to analyze the spectrum of infections caused by $S$. aureus and to study its antimicrobial resistance to commonly used antibiotics. Specimens from various clinical sites received in the laboratory for culture and sensitivity were processed as per standard techniques. Identification and susceptibility testing of $S$. aureus isolates were done using automated systems.

Results: A total of 234 S. aureus isolates were obtained during the study period. Males accounted for $70.1 \%$ (n=164) of patients with $S$. aureus infections. These patients were uniformly distributed across all age groups. S. aureus was most commonly isolated from pus and exudates (64.5\%) followed by respiratory specimens (20.5\%) and mainly cultured from the skin and soft tissue infections (56\%). Methicillin-resistant S. aureus (MRSA) accounted for $47 \%(\mathrm{n}=110)$ of isolates. Higher rates of susceptibility were noted for tetracycline (95.3\%), gentamicin $(85.4 \%)$, and trimethoprim/ sulfamethoxazole $(88 \%)$. Low susceptibility rate was seen for ciprofloxacin $(11.2 \%)$. Inducible clindamycin resistance was seen in $22.4 \%$ (n=50) isolates. Methicillin-sensitive $S$. aureus isolates were found to be more susceptible to non-beta lactam antibiotics than the methicillin-resistant isolates.

Conclusion: A high frequency of MRSA was found in our study. Regular surveillance of antimicrobial resistance profile of this most frequent pathogen is necessary to aid in providing appropriate empirical antibiotic therapy.

Keywords: Methicillin-resistant Staphylococcus aureus, Resistance, Skin and soft tissue infections, Staphylococcus aureus.

(C) 2017 The Authors. Published by Innovare Academic Sciences Pvt Ltd. This is an open access article under the CC BY license (http://creativecommons. org/licenses/by/4. 0/) DOI: http://dx.doi.org/10.22159/ajpcr.2017.v10i12.21851

\section{INTRODUCTION}

Staphylococcus aureus is an important bacterial pathogen responsible for a wide spectrum of clinical infections causing both communityacquired and healthcare-associated infections [1]. They cause skin and soft tissue infections (SSTIs) including impetigo, folliculitis, furunculosis, cutaneous abscesses, cellulitis, necrotizing fasciitis, and surgical site infections; pleuropulmonary infections; bone and joint infections and device-related infections. S. aureus is also one of the most frequent causes of infective endocarditis and bacteremia [1,2]. Toxinmediated illnesses, scalded skin syndrome, toxic shock syndrome, and food poisoning are also caused by S. aureus [1].

S. aureus frequently colonizes skin and mucosa and about $30 \%$ individuals being persistent nasal carriers. The extra nasal carriage sites include skin, perineum, and pharynx. S. aureus anterior nasal carriage is also associated with subsequent staphylococcal disease $[1,3]$.

Increasing resistance to different antibiotics including the glycopeptides among $S$. aureus isolates is a cause for concern. Methicillin-resistant S. aureus (MRSA), vancomycin-intermediate S. aureus (VISA), and vancomycin-resistant $S$. aureus (VRSA) are being increasingly encountered [4]. Significantly high rates of MRSA have been reported from India and various parts of the globe along with increasing reports of community-acquired MRSA infections [4-6]. Increasing resistance rates limit therapeutic options for $S$. aureus infections.

As $S$. aureus is one of the most frequent pathogens in a clinical setting and in view of its increasing resistance to common antibiotics, a prospective, observational study was conducted to analyze the spectrum of infections caused by $S$. aureus and its antibiotic susceptibility profile in a tertiary care teaching hospital setting.

\section{METHODS}

Following Institutional Ethics Committee approval, a prospective observational study was conducted of 4 months duration from January to April 2017, to study S. aureus isolates from various clinical specimens and to analyze their susceptibility pattern to various antibiotics. Duplicate isolates of $S$. aureus isolated from the same patient during the study period were excluded.

Specimens from various clinical sites received in the laboratory for culture and sensitivity were processed as per standard techniques [7]. The specimens were subjected to Gram stain and culture on 5\% sheep blood agar and MacConkey agar. Pus cells and bacteria were studied in the Gram stain. The inoculated culture plates were incubated at $37^{\circ} \mathrm{C}$ for $18-24$ hrs. S. aureus isolates were identified based on, colony morphology, Gram stain, catalase, slide coagulase test and by automated identification system - matrix-assisted laser desorption/ionization timeof-flight mass spectrometry (Vitek MS, bioMerieux Inc.). Antimicrobial susceptibility testing was done using the automated system (Vitek, bioMerieux Inc.). MRSA was identified with cefoxitin $(6 \mu \mathrm{g} / \mathrm{ml})$. Data were analyzed using standard statistical software - Statistical package for the social sciences (SPSS), version 17.0 (SPSS Inc., Chicago, Ill., USA). Quantitative variables were expressed as percentages.

\section{RESULTS}

A total of 234 S. aureus isolates were obtained during the study period from various infection sites. Males accounted for $70.1 \%(n=164)$ of patients with S. aureus infections. Patients with $S$. aureus infections were uniformly distributed across all age groups. Majority of these patients were from surgical departments (65.6\%) in the hospital. S. aureus was most commonly (64.5\%) isolated from pus and exudates (swabs, 
aspirated pus, and tissue) followed by respiratory specimens including sputum, endotracheal aspirates, bronchoalveolar lavage, throat swab, and nasal swab (20.5\%). S. aureus was mainly cultured from the SSTIs including surgical site infections (56\%) (Table 1).

Analyzing the resistance profile to various antimicrobials, we found $110(47 \%)$ of isolates to be MRSA. Among non-beta lactam antibiotics, higher rates of susceptibility were noted for tetracycline (95.3\%), gentamicin (85.4\%), and trimethoprim/sulfamethoxazole (88\%). Low susceptibility rates were seen for ciprofloxacin $(11.2 \%)$. Inducible clindamycin resistance was seen in $22.4 \%(\mathrm{n}=50)$ of $S$. aureus isolates $(n=223)$. All $S$. aureus isolates $(100 \%)$ were found to be sensitive to linezolid, vancomycin, and teicoplanin (Table 2). Lower rates of susceptibility were noted for ciprofloxacin (4.6\% vs. $16.9 \%$ ), clindamycin (62.6\% vs. $89.5 \%$ ), erythromycin (33.6\% vs. $64.5 \%)$, and gentamicin (73.4\% vs. $96 \%$ ) among MRSA isolates in comparison to methicillin-sensitive $S$. aureus (MSSA) isolates (Table 2). There was no significant difference in the infection profile between MSSA and MRSA isolates (Table 3).

Table 1: Clinical and demographic profile of patients with S. aureus infections $(n=234)$

\begin{tabular}{|c|c|}
\hline Characteristic & n (\%) \\
\hline \multicolumn{2}{|l|}{ Gender } \\
\hline Male & $164(70.1)$ \\
\hline Female & $70(29.9)$ \\
\hline Males:Females & $2.3: 1$ \\
\hline \multicolumn{2}{|l|}{ Age group (year) } \\
\hline$<1-20$ & $48(20.5)$ \\
\hline $21-40$ & $63(26.9)$ \\
\hline $41-60$ & $73(31.2)$ \\
\hline$>60$ & $50(21.4)$ \\
\hline Mean age (year) & $41 \pm 21.3$ \\
\hline Median age (year) & 43 \\
\hline Range (year) & $<1-90$ \\
\hline Patients admitted to hospital wards & $137(58.5)$ \\
\hline Patients managed as outpatients & $97(41.5)$ \\
\hline \multicolumn{2}{|c|}{ Patients distribution in clinical departments $(n=215)$} \\
\hline Surgical & $141(65.6)$ \\
\hline Medical & $74(34.4)$ \\
\hline \multicolumn{2}{|l|}{ Specimen types } \\
\hline Pus and exudates & $151(64.5)$ \\
\hline Respiratory specimens & $48(20.5)$ \\
\hline Blood and catheter tip & $26(11.1)$ \\
\hline Urine & $09(3.8)$ \\
\hline \multicolumn{2}{|l|}{ Infection site } \\
\hline Skin and soft tissue & $131(56)$ \\
\hline Lower respiratory tract & $26(11.1)$ \\
\hline Bloodstream & $26(11.1)$ \\
\hline Upper respiratory tract & $22(9.4)$ \\
\hline Otogenic & $14(6)$ \\
\hline Urinary tract & $09(3.8)$ \\
\hline Bone and joint & $06(2.6)$ \\
\hline
\end{tabular}

S. aureus: Staphylococcus aureus

\section{DISCUSSION}

$S$. aureus continues to be a significant human pathogen commonly isolated from various clinical specimens. A high number of $S$. aureus isolates were obtained from males (70.1\%). Male gender and extreme of ages are found to be at increased risk for infections caused by S. aureus by population-based studies [2]. However, S. aureus infections were evenly distributed across various age groups in our analysis.

In our study, $S$. aureus was most commonly isolated from SSTIs (56\%). Ray et al., in their retrospective analysis found $S$. aureus as the etiological agent in $81 \%$ of positive cultures from SSTI specimens [8]. Globally, S. aureus is recognized as the most frequent pathogen isolated from SSTIs [9]. Apart from S. aureus, Pseudomonas aeruginosa, Enterococcus spp., Escherichia coli, and beta-hemolytic Streptococci are other important causes of SSTIs [8]. Most SSTIs occur when the innate immune barrier, the skin is breached by trauma or surgery. Infections may also spread from a distant site [10].

Analyzing the antimicrobial susceptibility data of $S$. aureus isolates, high rate of resistance to ciprofloxacin was seen (88.8\%). Bouchiat et al., in their analysis of 92 S. aureus clinical isolates, found $70.6 \%(n=65)$ of them to be resistant to ciprofloxacin [11]. Gade and Qazi have reported $57.6 \%(n=144)$ isolates of $S$. aureus to be ciprofloxacin resistant [12]. High rates of quinolone resistance have also been noted earlier by Metri et al. among S. aureus isolates $(n=137)$ [13]. Use of quinolones for various infections leads to the selection of resistant mutants among the $S$. aureus colonizing flora which acts as a reservoir for future infections, and quinolone resistance are more predominantly noted in MRSA isolates [14].

Lower rate of resistance to trimethoprim/sulfamethoxazole was noted $(12 \%)$ in our study. Trimethoprim-sulfamethoxazole can be used for the treatment of $S$. aureus infections such as uncomplicated SSTI [15-17]. Majority of our S. aureus isolates (77.1\%) were sensitive to clindamycin, an important alternative to a beta-lactam antibiotic for $S$. aureus SSTIs $[18,19]$. Inducible clindamycin resistance $\left(\mathrm{iMLS}_{\mathrm{B}}\right.$ ) was seen in $22.4 \%$ of our $S$. aureus isolates. Prabhu et al. and Deotale et al. have reported lower rates of $10.5 \%(n=20)$ and $14.5 \%(n=36)$, respectively, for iMLS $_{\mathrm{B}}[18,19]$.

In our study, $97.8 \%$ of $S$. aureus isolates were resistant to benzylpenicillin. This is because the majority of the clinical isolates of $S$. aureus produce beta-lactamase enzyme which inactivates the penicillin antibiotic [7]. The rate of MRSA was high in our study (47\%). Varying rates of MRSA prevalence have been reported across different centers [11]. A multi-centric study by the Indian Network for Surveillance of Antimicrobial Resistance group involving 15 tertiary care centers found a MRSA prevalence rate of $41 \%(n=10769)$ [5]. Bouchiat et al. found 52.2\% $(\mathrm{n}=48)$ isolates of $S$. aureus to be methicillin-resistant [11]. Thati et al. recorded $79.6 \%(n=285)$ S. aureus isolates to be methicillin-resistant [20]. Other studies have found lower rates of MRSA prevalence [21,22]. Dilnessa and Bitew have reported MRSA rates of $17.5 \%(n=34)$ in their analysis of 194 S. aureus clinical isolates [21]. In an evaluation of antibiotic resistance profile of $S$. aureus isolates over several years by Ragbetli et al., $21 \%$ of them were methicillin-resistant [22]. S. aureus is commonly transmitted by skinto-skin contact with either a colonized or infected individual. Indirect contact through fomites also plays a role in the transmission of MRSA. The 5 Cs for MRSA spread proposed by Centers for Disease Control and Prevention, USA includes crowding, frequent skin-to-skin contact, compromised skin integrity, contaminated items and surfaces, and lack of cleanliness [6]. We did not find a significant difference in infection profile between MRSA and MSSA isolates, mirroring the finding of Dilnessa and Bitew [21]. MRSA strains were found to be more resistant to non-beta lactam antibiotics compared to MSSA (Table 2), similar to findings of earlier studies $[5,11,12]$. Infection control practices need to be further strengthened and complemented with judicious antimicrobial prescribing to reduce the burden of MRSA infections. 
Table 2: Susceptibility pattern of S. aureus isolates to various antibiotics

\begin{tabular}{llll}
\hline Antibiotic (No. of isolates tested) & $\begin{array}{l}\text { Susceptible isolates } \\
\text { among MSSA n (\%) }\end{array}$ & $\begin{array}{l}\text { Susceptible isolates } \\
\text { among MRSA n (\%) }\end{array}$ & $\begin{array}{l}\text { Total susceptible isolates } \\
\text { of S. aureus } \mathbf{n} \text { (\%) }\end{array}$ \\
\hline Benzylpenicillin (225) & $5(4.1)$ & 0 & $5(2.2)$ \\
Oxacillin (234) & $124(100)$ & 0 & $124(53)$ \\
Ciprofloxacin (232) & $21(16.9)$ & $5(4.6)$ & $26(11.2)$ \\
Gentamicin (233) & $119(96.0)$ & $80(73.4)$ & $199(85.4)$ \\
Erythromycin (231) & $80(64.5)$ & $36(33.6)$ & $116(50.2)$ \\
Clindamycin (231) & $111(89.5)$ & $67(62.6)$ & $178(77.1)$ \\
Tetracycline (232) & $121(97.6)$ & $100(92.6)$ & $221(95.3)$ \\
Trimethoprim/Sulfamethoxazole (233) & $113(91.1)$ & $92(82.4)$ & $205(88)$ \\
Rifampicin (229) & $122(100)$ & $104(97.2)$ & $226(98.7)$ \\
Daptomycin (225) & $121(100)$ & $103(99.0)$ & $224(99.6)$ \\
Linezolid (230) & $122(100)$ & $108(100)$ & $230(100)$ \\
Vancomycin (230) & $122(100)$ & $108(100)$ & $230(100)$ \\
Teicoplanin (229) & $122(100)$ & $107(100)$ & $229(100)$ \\
\hline
\end{tabular}

*MSSA: Methicillin-sensitive Staphylococcus aureus, MRSA: Methicillin-resistant Staphylococcus aureus, S. aureus: Staphylococcus aureus

Table 3: Distribution of MSSA and MRSA isolates in various infections

\begin{tabular}{lll}
\hline Infection type (Number of isolates) & Isolates of MSSA n=124 (\%) & Isolates of MRSA n=110 (\%) \\
\hline Skin and soft tissue infections (131) & $71(54.2)$ & $60(45.8)$ \\
Lower respiratory tract infections (26) & $11(42.3)$ & $15(57.7)$ \\
Bloodstream infections (26) & $10(38.5)$ & $16(61.5)$ \\
Upper respiratory tract infections (22) & $12(54.5)$ & $10(45.4)$ \\
Otogenic infections (14) & $09(64.3)$ & $05(35.7)$ \\
Urinary tract infections (09) & $06(66.7)$ & $03(33.3)$ \\
Bone and joint infections (06) & $05(83.3)$ & $01(16.7)$ \\
Total & $124(100)$ & $110(100)$ \\
\hline
\end{tabular}

*MSSA: Methicillin-sensitive Staphylococcus aureus, MRSA: Methicillin-resistant Staphylococcus aureus, S. aureus: Staphylococcus aureus

All S. aureus isolates including MRSA were found to be sensitive to vancomycin, linezolid, and teicoplanin. There were no cases of VISA or VRSA in our study. All isolates of MSSA and MRSA were sensitive to vancomycin with a median MIC of $0.5 \mu \mathrm{g} / \mathrm{ml}$. A clinical isolate of VISA was initially reported from Japan in 1997, and the first report of VRSA was from the United States in 2002 [23]. Since then, even though less frequent, studies from India and globally are increasingly reporting the occurrence of both VISA and VRSA among clinical isolates of $S$. aureus [20,24,25]. Large multi-centric studies are needed to analyze the prevalence and implications of VISA and VRSA in the clinical setting. It is estimated that $<1 \%$ of $S$. aureus isolates are linezolid-resistant [26].

We did not analyze the antimicrobial prescription data and prognosis of patients with S. aureus infections. Analysis of risk factors for MRSA infections and colonization status was not done.

\section{CONCLUSION}

S. aureus was more frequently associated with SSTIs. A high frequency of MRSA was found in our study. Generating local data on antimicrobial resistance among the $S$. aureus isolates will help in choosing appropriate empirical antibiotic therapy based on the clinical presentation

\section{REFERENCES}

1. Tong SY, Davis JS, Eichenberger E, Holland TL, Fowler VG Jr. Staphylococcus aureus infections: Epidemiology, pathophysiology, clinical manifestations, and management. Clin Microbiol Rev 2015;28(3):603-61.

2. Que YA, Moreillon P. Staphylococcus aureus (Including Staphylococcal Toxic Shock Syndrome). In: Bennett JE, Dolin R, Blaser MJ, editors. Mandell, Douglas, and Bennett's Principles and Practice of Infectious Diseases. $8^{\text {th }}$ ed. Philadelphia: Elsevier, Saunders; 2015. p. 2237-71.

3. Wertheim HF, Melles DC, Vos MC, van Leeuwen W, van Belkum A, Verbrugh HA, et al. The role of nasal carriage in Staphylococcus aureus infections. Lancet Infect Dis 2005;5(12):751-62.
4. Chen CJ, Huang YC. New epidemiology of Staphylococcus aureus infection in Asia. Clin Microbiol Infect 2014;20(7):605-23.

5. Indian Network for Surveillance of Antimicrobial Resistance (INSAR) group, India. Methicillin resistant Staphylococcus aureus (MRSA) in India: Prevalence \& susceptibility pattern. Indian J Med Res 2013;137(2):363-9.

6. Kale P, Dhawan B. The changing face of community-acquired methicillin-resistant Staphylococcus aureus. Indian J Med Microbiol 2016;34(3):275-85.

7. Baird D. Staphylococcus: Cluster forming gram positive cocci. In: Collee JG, Fraser AG, Marmion BP, Simmons A, editors. Mackie and McCartney Practical Medical Microbiology. $14^{\text {th }}$ ed. London: Churchill Livingstone; 2012. p. 245-61.

8. Ray GT, Suaya JA, Baxter R. Incidence, microbiology, and patient characteristics of skin and soft-tissue infections in a U.S. population: A retrospective population-based study. BMC Infect Dis 2013;13:252.

9. McCaig LF, McDonald LC, Mandal S, Jernigan DB. Staphylococcus aureus-associated skin and soft tissue infections in ambulatory care. Emerg Infect Dis 2006;12(11):1715-23.

10. Ramakrishnan K, Salinas RC, Agudelo Higuita NI. Skin and soft tissue infections. Am Fam Physician 2015;92(6):474-83.

11. Bouchiat C, El-Zeenni N, Chakrakodi B, Nagaraj S, Arakere G, Etienne J. Epidemiology of Staphylococcus aureus in Bangalore, India: Emergence of the ST217 clone and high rate of resistance to erythromycin and ciprofloxacin in the community. New Microbes New Infect 2015;7:15-20.

12. Gade ND, Qazi MS. Fluoroquinolone therapy in Staphylococcus aureus infections: Where Do We Stand? J Lab Physicians 2013;5(2):109-12.

13. Metri BC, Peerapur BV, Jyothi P. Drug resistance patterns of clinical isolates of Staphylococcus aureus in tertiary care center of south India. Int J Pharm Pharm Sci 2015;7(7):70-2.

14. Lowy FD. Antimicrobial resistance: The example of Staphylococcus aureus. J Clin Invest 2003;111(9):1265-73

15. Paul M, Bishara J, Yahav D, Goldberg E, Neuberger A, GhanemZoubi N, et al. Trimethoprim-sulfamethoxazole versus vancomycin for severe infections caused by methicillin resistant Staphylococcus aureus: Randomised controlled trial. BMJ 2015;350:h2219.

16. Cadena J, Nair S, Henao-Martinez AF, Jorgensen JH, Patterson JE, Sreeramoju PV. Dose of trimethoprim-sulfamethoxazole to treat skin and skin structure infections caused by methicillin-resistant Staphylococcus 
aureus. Antimicrob Agents Chemother 2011;55(12):5430-2.

17. Liu C, Bayer A, Cosgrove SE, Daum RS, Fridkin SK, Gorwitz RJ, et al. Clinical practice guidelines by the infectious diseases society of America for the treatment of methicillin-resistant Staphylococcus aureus infections in adults and children. Clin Infect Dis 2011;52(3):e18-55.

18. Prabhu K, Rao S, Rao V. Inducible Clindamycin Resistance in Staphylococcus aureus isolated from clinical samples. J Lab Physicians 2011;3(1):25-7.

19. Deotale V, Mendiratta DK, Raut U, Narang P. Inducible clindamycin resistance in Staphylococcus aureus isolated from clinical samples. Ind J Med Microbiol 2010;28(2):124-6.

20. Thati V, Shivannavar CT, Gaddad SM. Vancomycin resistance among methicillin resistant Staphylococcus aureus isolates from intensive care units of tertiary care hospitals in Hyderabad. Indian J Med Res 2011;134(5):704-8.

21. Dilnessa T, Bitew A. Prevalence and antimicrobial susceptibility pattern of methicillin resistant Staphylococcus aureus isolated from clinical samples at Yekatit 12 Hospital Medical College, Addis Ababa, Ethiopia. BMC Infect Dis 2016;16:398.

22. Ragbetli C, Parlak M, Bayram Y, Guducuoglu H, Ceylan N. Evaluation of antimicrobial resistance in Staphylococcus aureus isolates by years. Interdiscip Perspect Infect Dis 2016;2016:9171395.

23. Sangappa M, Thiagarajan P. Methicillin resistant Staphylococcus aureus: Resistance genes and their regulation. Int J Pharm Pharm Sci 2012;4 Suppl 1:658-67.

24. Holmes NE, Johnson PD, Howden BP. Relationship between vancomycin-resistant Staphylococcus aureus, vancomycinintermediate $S$. aureus, high vancomycin MIC, and outcome in serious S. aureus infections. J Clin Microbiol 2012;50(8):2548-52.

25. Loomba PS, Taneja J, Mishra B. Methicillin and vancomycin Resistant $S$. aureus in Hospitalized Patients. J Glob Infect Dis 2010;2(3):275-83.

26. Gu B, Kelesidis T, Tsiodras S, Hindler J, Humphries RM. The emerging problem of linezolid-resistant Staphylococcus. J Antimicrob Chemother 2013;68(1):4-11 